\title{
Evaluation of High Field Responses of Fine Scale Piezoelectric Fibres
}

\author{
L. J. NELSON, ${ }^{1}$ C. R. BOWEN, ${ }^{1}$ R. STEVENS, ${ }^{1}$ \\ M. CAIN ${ }^{2}$ and M. STEWART ${ }^{2}$ \\ ${ }^{1}$ Materials Research Centre, University of Bath, Bath, BA2 7AY, England \\ ${ }^{2}$ Materials Centre, National Physical Laboratory, Teddington, Middlesex, \\ TW15 IUY, England
}

(Received August 8, 2003; in final form January 5, 2004)

\begin{abstract}
A methodology for the measurement of high field strain and polarisation responses of fine scale piezoelectric fibres is presented. Using analytical models, the response of the fibres is extracted from measurements made on 1-3 composites. Analytical modelling shows that composites with a high fibre volume fraction are desirable for accurate prediction of the fibres response. Poor model response at low fibre volume fractions is attributed to non-linear material properties, and deviation from the model assumptions.
\end{abstract}

Keywords: Piezoelectric; fibres; high field; strain; polarisation; composite

\section{INTRODUCTION}

Fine scale piezoelectric fibres are finding uses in novel geometry composites for actuator and sensor applications. In actuator mode these composites, which are typically referred to as Active Fibre Composites (AFCs) [1], are driven at high electric fields $\left(1-2 \mathrm{kVmm}^{-1}\right)$ to induce maximum stresses and strains. Therefore, it is important that the high field properties of the active fibres are characterised. Analytical models exist which allow the low field properties of fibres to be predicted from 1-3 composite properties [2]. This research extends this model to enable the high field polarisation and strain responses of piezoelectric fibres to be measured. In the first section the analytical models are introduced. In derivation of both the strain and polarisation equations it is assumed that the fibres are sufficiently fine such that the fibre strain and the polymer matrix strain in the 3-direction (fibre direction) are identical and equal the composite strain (the iso-strain assumption). The influence of this assumption is discussed with reference to the model validity. The effect of non-linear material properties, such as $d_{33}$, is also investigated. 
The proposed methodology is validated in the second section by comparing predicted results with experimental data. The measured responses of the piezoelectric fibre are presented in the final section.

\section{ANALYTICAL MODELLING}

\subsection{Strain-Field Response}

When subjected to an electric field the poled fibres in a 1-3 composite will strain. The matrix piezoelectric strain is zero since it is a passive polymer that exhibits no piezoelectric behaviour. Since the matrix is assumed to be perfectly bonded to the fibres, it acts to clamp, and hence reduce, the fibre strain. The piezoelectric strain of the fibres at electric field $E, S_{\text {piezo }}^{f}(E)$, is related to the composite strain, $\bar{S}(E)$, through:

$$
\bar{S}(E)=S_{\text {piezo }}^{f}(E)\left[\frac{v s_{11}}{v s_{11}+(1-v) s_{33}^{E}}\right]
$$

In which $s_{11}$ and $s_{33}^{E}$ are the compliances of the matrix and fibre respectively (assumed to be independent of electric field) and $v$ is the fibre volume fraction. This equation is obtained by multiplying the analytical expression for 13 composite $d_{33}$ (strain per unit applied field), developed by Smith [2], by the electric field. Equation (1) allows the fibre piezoelectric strain-field response to be extracted from measurements of composite strain-field responses, provided the compliance and volume fraction of each phase is known.

It is probable that the iso-strain assumption is not be strictly observed in all composites. Deviation from iso-strian conditions occurs when the fibre volume fraction and matrix stiffness reduce, and as fibre aspect ratio (diameter/length) increases [3]. In such cases, fields applied in the poling direction will cause fibres to protrude from the composite surface, while fields opposing the poling direction will result in fibres contracting into the composite. Composite strains measured using surface contact techniques (as in this research) will be influenced by this effect.

\subsection{Polarisation-Field Response}

The composites polarisation at electric field $E, \bar{P}(E)$, can be expressed as a combination of the polarisations developed from three sources:

$$
\bar{P}(E)=\underbrace{\left[v P^{f}(E)\right]}_{\begin{array}{c}
\text { Fibre contribution } \\
\left(P_{\text {fibre }}\right)
\end{array}}+\underbrace{\left[(1-v) P^{m}(E)\right]}_{\begin{array}{c}
\text { Matrix contribution } \\
\left(P_{\text {matrix }}\right)
\end{array}}-\underbrace{\left[\frac{v(1-v)\left(d_{33}(E)\right)^{2} E}{v s_{11}+(1-v) s_{33}^{E}}\right]}_{\begin{array}{c}
\text { Clamping contribution } \\
\left(P_{\text {clamp }}\right)
\end{array}}
$$


In addition to the polarisation developed by the fibre, $P^{f}(E)$, and matrix, $P^{m}(E)$, a polarisation is developed from active fibre clamping. As a field is applied to the composite the fibres are caused to strain, but the passive matrix partially clamps the full extension. This clamping causes the fibres to develop a charge via their $d_{33}$ effect, which reduces the overall polarisation measured. This is quantified as the 'clamping contribution' $\left(P_{\text {clamp }}\right)$ in Eq. (2), in which $d_{33}(E)$ is the fibre $d_{33}$ coefficient at electric field $E$. Equation (2) is obtained by multiplying the analytical expression for 1-3 composite permitivity, developed by Smith [2], by the electric field. The importance of this equation is that it enables the polarisation response of the fibres to be extracted from the 1-3 composite response, provided the volume fraction and stiffness' of each phase, the fibre $d_{33}$ at electric field $E$, and the matrix permitivity are known since $P^{m}(E)$ is simply the product of the matrix permitivity and electric field.

At relatively high electric fields $\left(0.5 \mathrm{kVmm}^{-1}\right)$ it is not uncommon for the $d_{33}$ coefficient of a soft PZT to increase by $300 \%$ of its quoted low field value (600 to $2000 \mathrm{pCN}^{-1}$ ) [4]. If the non-linearity of the fibre $d_{33}$ is not quantified, significant errors could be introduced into the predicted response, due to miscalculation of the clamping contribution. Figure 1 shows the contribution to the composite polarisation $\left(P_{\text {comp }}\right)$ arising from the

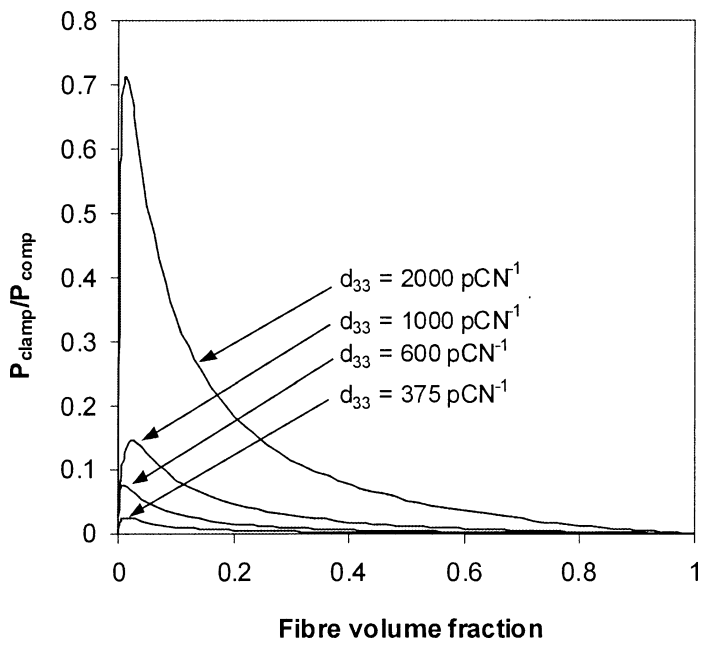

FIGURE 1 Contribution to the total polarisation of a 1-3 composite from fibre clamping ( $\left.P_{\text {clamp }} / P_{\text {comp }}\right)$ for four different fibre $d_{33}$ constants as a function of fibre volume fraction. 
fibre clamping ( $\left.P_{\text {clamp }}\right)$, as a function of fibre volume fraction and fibre $d_{33}$ coefficient.

Figure 1 shows that at high fibre volume fractions $(v>0.6)$ the matrix exerts very little restraining force on the fibres, and the contribution to the total polarisation from the clamping effect is small. As the fibre volume fraction decreases the matrix begins to exert a stronger clamping effect and the contribution increases. A maximum contribution is achieved at approximately $2 \%$ fibres. On reducing the volume fraction below $2 \%$ the fibre clamping continues to increase, but the area of fibre from which the charge is generated decreases such that the overall contribution diminishes. The effect of increasing the fibre $d_{33}$ coefficient is shown in Fig. 1 to highlight the effect of high field testing. If it is assumed that fibre $d_{33}$ remains at its low field value $\left(375 \mathrm{pCN}^{-1}\right)$ during high field testing, then polarisations predicted using Eq. (2) will be underestimated. The degree of underestimation will be larger for low volume fraction composites and will increase as the fibre $d_{33}$ increases with increasing field. Deviation from the iso-strain assumption will result in incomplete stress transfer from the matrix to the fibres. Thus the contribution to the composite polarisation from the clamping effect will be reduced. In these cases polarisations predicted using Eq. (2) will be overestimated. These observations, and Fig. 1, show that the fibre polarisation response can be estimated using the low field value of $d_{33}$ in Eq. (2) without the introduction of significant errors if high volume fraction composites are tested $(v>0.6)$ since $P_{\text {clamp }} / P_{\text {comp }}$ is small.

\section{MODEL VALIDATION}

Composites with 1-3 connectivity, and spanning a volume fraction range from 0.02 to 0.72 , were manufactured using PZT-5A fibres $250 \mu \mathrm{m}$ in diameter [5] using techniques described in reference [6]. To validate the analytical models, the strain and polarisation response of the composites was measured using a system designed at the National Physical Laboratory [7]. Values of saturation strain and saturation polarisation, simultaneously measured from composites subjected to a sinusoidal field of $\pm 2.0 \mathrm{kV} \mathrm{mm}^{-1}$, were used with Eqs. (1) and (2) to extract the fibre saturation strain and polarisation values respectively. Volume fractions were measured using image analysis techniques while fibre and matrix properties $\left(s_{33}^{E}, d_{33}\right.$ and $\left.s_{11}\right)$ were taken from reference [6]. The fibre saturation strain and polarisation values, extracted using Eqs. (1) and (2), are plotted against the fibre volume fraction of the composite in Fig. 2. For the model to accurately predict the fibre response, 


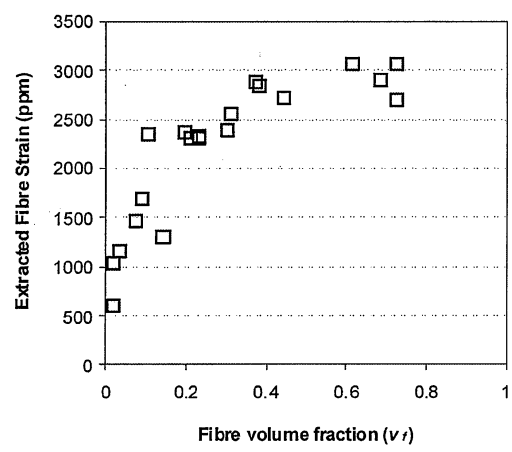

(a)

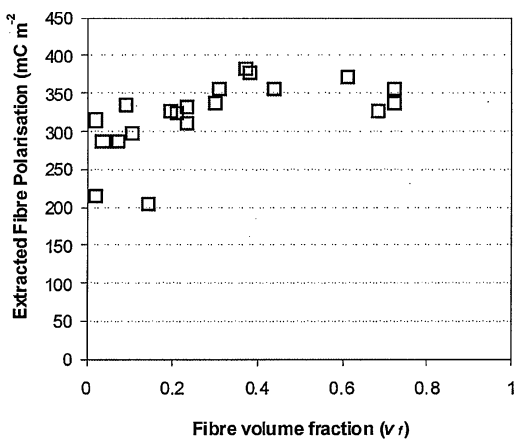

(b)

FIGURE 2 Extracted fibre values of (a) saturation strain and (b) saturation polarisation for a cyclic field of $2.0 \mathrm{kVmm}^{-1}$. Extracted values are plotted against the volume fraction of the composite from which they were extracted.

a graph of extracted fibre strain or polarisation versus fibre volume fraction should be constant and form a horizontal line, the average value of which represents the fibre strain for that field level.

From Fig. 2 it is clear that the model is incapable of predicting the fibre responses over the entire volume fraction range, especially the strain response (Fig. 2(a)). It has been shown in Fig. 1 that errors associated with non-linear material properties are highest at low volume fractions. It is also known that and deviation form the iso-strain assumption is largest at low

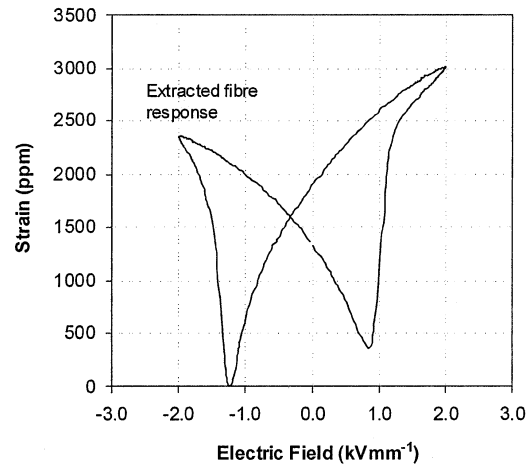

(a)

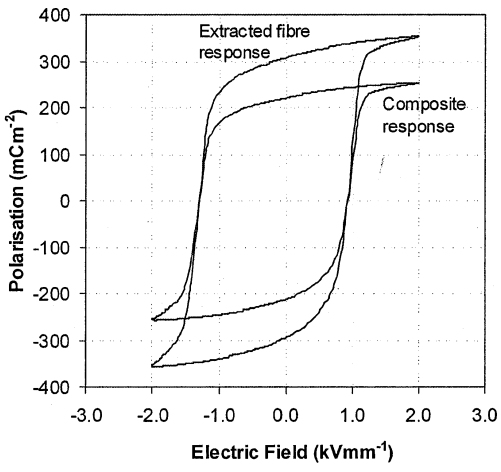

(b)

FIGURE 3 Fibre responses of (a) strain and (b) polarisation extracted from measurements made on 1-3 composites. Composite fibre volume fraction is 0.72 . 
volume fractions [3]. These two effects can be attributed to the observed behaviour. It can be concluded that fibre responses are more likely to be predicted precisely at high volume fractions. Figure 3(a) and (b) show plots of the strain-field and polarisation-field responses of a composite, and the fibre response extracted using Eqs. (1) and (2). Since the response is taken from a high volume fraction composite $(v=0.72)$ the composite strain is only reduced by approximately $1.7 \%$ and is thus omitted for clarity in Fig. 3(a).

\section{CONCLUSIONS}

This paper has proposed a method for extracting the strain and polarisation response of fine scale fibres from measurements performed on 1-3 composites. This is a useful technique for comparing the strain-field and polarisation-field responses of different fibres and enables the selection of appropriate fibres for AFC manufacture. The effect of non-linear material properties was discussed and found to have implications for the polarisation response, in which the clamping contribution to the composites response becomes significant and can lead to errors at low fibre volume fractions and high fields. Validation of the models using experimental data revealed that significant deviation occurs in the strain response at low volume fractions. This is thought to be associated with composites not satisfying the iso-strain assumption of the analytical model and is being investigated in future work. For the accurate extraction of fibre responses it is advisable to test high volume fraction composites $(v>0.6)$, in which non-linear material properties and deviation from the iso-strain assumption become less influential.

\section{ACKNOWLEDGEMENTS}

The author wishes to thank the University of Bath and the National Physical Laboratory for funding this research.

\section{REFERENCES}

[1] A. Bent, Active fiber composites for structural actuation. PhD Thesis. Massachusetts Institute of Technology (AMSL). (1997).

[2] W. A. Smith, "Modelling 1-3 composite piezoelectrics: Hydrostatic response," IEEE Trans Ultrason, Ferroelec, Freq Contr. 40(1), 40-47 (1993).

[3] W. Cao, Q. M. Zang, and L. E. Cross, "Theoretical study on the static performance of 
piezoelectric ceramic-polymer composites with 1-3 connectivity," J. Appl. Phys. 72(12), 5814-5821 (1992).

[4] D. Hall, "Nonlinearity in piezoelectric ceramics," J. Mater. Sci. 36, 4575-4601 (2001).

[5] Smart Material Corp.: [http://www.smart-material.com/]

[6] L. Nelson, C. Bowen, R. Stevens, M. Cain, and M. Stewart, "Modelling and measurement of piezoelectric fibres and interdigitated electrodes for the optimisation of piezofibre composites," SPIEs 10th Int Symp on Smart Structures and Materials, San Diego (CA). Paper 5053-73 (March 2003).

[7] M. Stewart, M. G. Cain, and D. A. Hall, Ferroelectric hysteresis measurement and analysis. Report CMMT (A) 152, National Physical Laboratory, Teddington. (May 1999). 